\title{
Visualizing Interfirm Comparison
}

\author{
EM VERMEULEN
}

\section{J SPRONK}

\author{
D VAN DER WIJST \\ Erasmus University Rotterdam, The Netherlands
}

(Received August 1993; in revised form May 1994)

\begin{abstract}
In this paper, we present a new way to visualize the results obtained with interfirm comparison. It will be shown how these results can be visualized even if the explanatory variable is explained by more than one firm characteristic. As a result, the new visualization method enables us to see at a single glance: which firm has the highest explanatory variable, what the expected value of the explanatory variable is given the firm's characteristics, what the corresponding values of its competitors are, and to what extent the different firm characteristics contribute to the firm's expected value of the explanatory variable. The method is illustrated by a numerical example concerning Dutch retail industries.
\end{abstract}

Key words-interfirm comparisons, industry analysis, risk analysis, linear models

\section{INTRODUCTION}

THE AIM OF THIS PAPER is to present a new way to visualize interfirm comparison. Modern interfirm comparison techniques as described by Van der Wijst [2] make use of regression analysis. In such an analysis the variable of interest is in general explained by more than one firm characteristic, which serve as regressors, i.e. as explanatory variables.

However, visualization of this method was possible only when one regressor was used. In this paper, we present a way to visualize the results of interfirm comparison when more than one regressor is used.

The method will be illustrated by a numerical example. However, instead of comparing financial ratios of firms, we compare the risks firms run. Risk is measured by means of a so-called multi-factor model, which is extensively described in [1]. The resulting risk measure contains the sensitivities for several external risk factors, such as the business cycle and the interest rate. In this paper, we restrict ourselves to a comparison of the sensitivity for the business cycle.

The organization of this paper is as follows. Section 2 describes previous research on interfirm comparison as carried out by Van der Wijst [2]. Section 3 shows the new visualization method. To illustrate this, section 4 provides a numerical example. Section 5 summarizes and concludes.

\section{INTERFIRM COMPARISON}

In this section, we will briefly describe how Van der Wijst [2] performs interfirm comparison making use of multi-regressor regression techniques. It will also be shown how in the oneregressor case results obtained with the method can be visualized neatly.

Generally speaking, the purpose of interfirm comparison is to gain insight into the weak- 
nesses and strengths of a firm. Strong and weak points are usually ascertained by comparing certain financial ratios of a firm with the industry average (or median) ratio.

However, these average industry ratios are nearly always too general, and, consequently, not appropriate as a comparison measure. Notably, the 'normal' value of the ratio usually also depends on firm characteristics that differ within industries, such as the products or services provided by the firm, or the assets employed. As an example, one can think of the 'normal' value of the debt ratio, which is probably higher for firms that own their equipment as opposed to those that lease it.

To correct for these differences in firm characteristics or, in other words, to distinguish between a firm's performance and its characteristics, Van der Wijst [2] makes use of regression analysis and explains the ratio by firm characteristics as follows:

$$
y_{i}=\beta_{0}+\beta_{1} \cdot x_{i 1}+\cdots+\beta_{k} \cdot x_{i k}+\epsilon_{i}
$$

where $i$ stands for the individual firm, $y_{i}$ denotes the firm's ratio, $x_{i j}$ is the value of firm characteristic $j$ of firm $i, \beta_{j}$ are the parameters to be estimated, and $\epsilon_{i}$ is an error term.

After estimating the parameters $\beta$, the expected 'normal' ratio for a firm with characteristics $x_{i l}, \ldots, x_{i k}$ is calculated as follows:

$$
\hat{y}_{i}=\hat{\beta}_{0}+\hat{\beta}_{1} \cdot x_{i 1}+\cdots+\hat{\beta}_{k} \cdot x_{i k}
$$

where $\hat{y}_{i}$ stands for the firm's expected 'normal' ratio, and represents the expected ratio value of a firm with characteristics of firm $i$.

If only one firm characteristic is used to explain the ratio, we will speak of the one-regressor case. The corresponding expression, then, is:

$$
\hat{y}_{i}=\hat{\beta}_{0}+\hat{\beta}_{1} \cdot x_{\mathrm{n}} .
$$

This relation can be visualized in a two-dimensional figure, such as Fig. 1. In this figure, the horizontal axis refers to the value of $x_{i 1}$ and the vertical axis to the corresponding value of $\hat{y}_{i}$. The firms are positioned in accordance with their $y_{i}$ and $x_{i 1}$ values. Now, it can be seen that for example firm A has a $y$-value which is higher than expected and firm B has a $y$-value which is lower than expected. This way to visualize the results obtained with interfirm comparison in the one-regressor case is drawn from [2].

However, this kind of visualization becomes difficult in the two-regressor case, i.e. if more than one firm characteristic is used to explain the ratio. A three-dimensional figure would have to be used. But when three firm characteristics are used to explain the financial ratio, even this is impossible.

Visualization of the technique is important, because the results of an interfirm comparison analysis have to be presented to managers, with a usually limited background in analytical techniques. Yet, they have to accept and, thus, first,

\section{Y-axis}

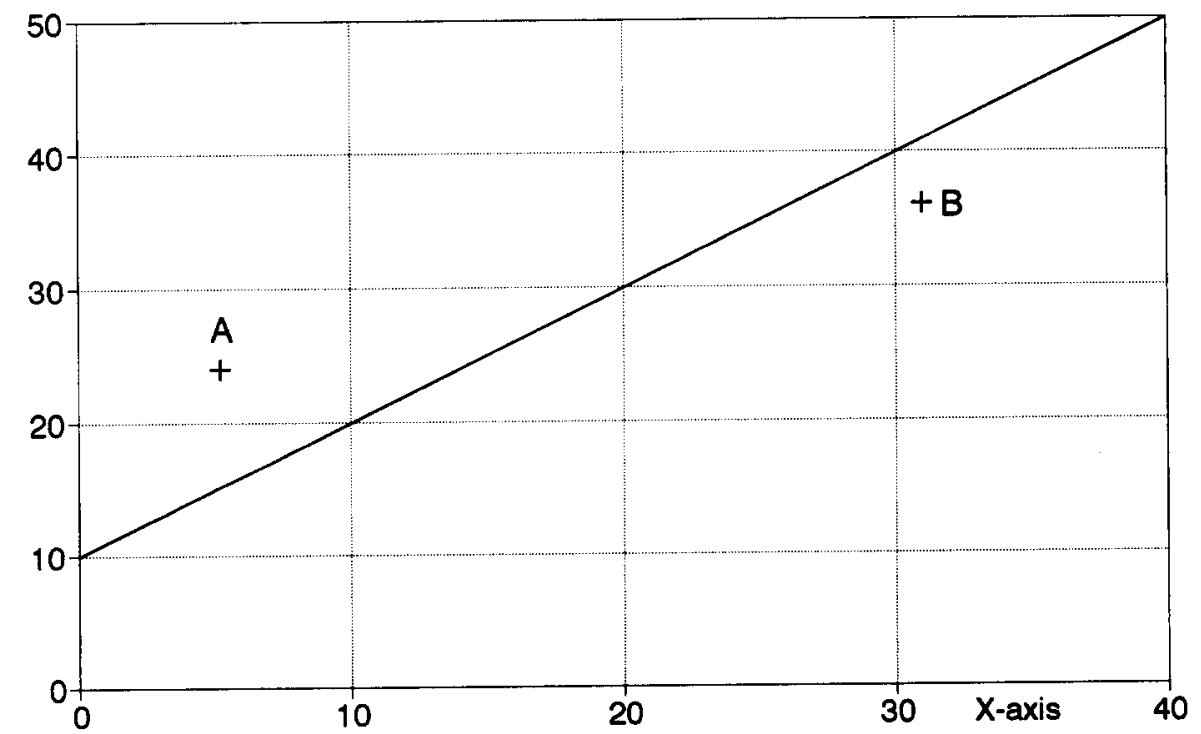

Fig. 1. Interfirm comparison in the one-regressor case. 


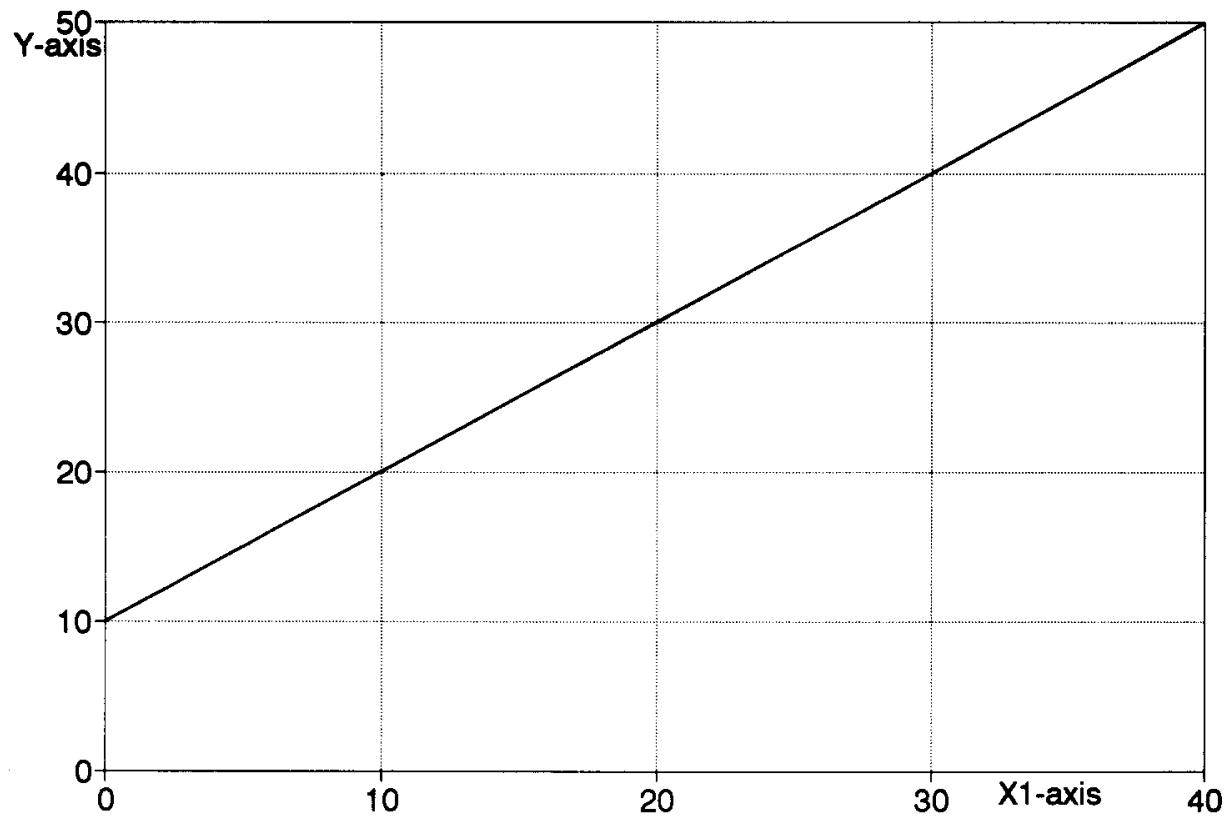

Fig. 2. Plot of $Y$ vs $X 1$.

understand the results, if the interfirm comparison is to be useful at all.

\section{MULTI-REGRESSOR INTERFIRM COMPARISON}

In this section, it will be shown how a clear visualization of interfirm comparison techniques is also possible when more than one regressor is used to explain the ratio.

The method is based on the use of more than one $x$-axis. To illustrate this, we will start with a simple two-regressor case, which is represented in the following expression.

$$
\hat{y}_{i}=\hat{\beta}_{0}+x_{i 1} \cdot \hat{\beta}_{1}+x_{i 2} \cdot \hat{\beta}_{2} \text {. }
$$

Suppose that $\hat{\beta}_{0}=10, \hat{\beta}_{1}=1$, and $\hat{\beta}_{2}=2$. First, assume that $x_{i 2}=0$. Figure 2 presents the relation between $\hat{y}_{i}$ and $x_{i 1}$ in this case. The slope of the line is $\beta_{1}=1$. Possible values for $x_{i 1}$ range from zero to $C$, which is chosen to be 40 . Next, assume that $x_{i 2} \neq 0$. Then, one unit of $x_{i 2}$ has the same influence on $\hat{y}_{i}$ as $\left(\hat{\beta}_{2} / \hat{\beta}_{1}\right)$, i.e. $(2 / 1)=2$, units of $x_{i 1}$.' Conversely, one unit of $x_{i 1}$ has the same influence as $\left(\hat{\beta}_{1} / \hat{\beta}_{2}\right)$, i.e. $(1 / 2)=0.5$, units of $x_{i 2}$. Hence, $C$ units of $x_{i 1}$ have the same influence on $\hat{y}_{i}$ of $\left(\hat{\beta}_{1} / \hat{\beta}_{2}\right) * C$, i.e.

'Proof: If $x_{i 2}$ increases with 1 , then $\hat{y}_{i}$ increases with $\Delta x_{i 2} \cdot \hat{\beta}_{2}=1 \cdot \hat{\beta}_{2}=\hat{\beta}_{2} ;$ if $x_{i 1}$ increases with $\left(\hat{\beta}_{2} / \hat{\beta}_{1}\right)$, then $y_{i}$ increases with $\Delta x_{i 1} \cdot \hat{\beta}_{1}=\left(\hat{\beta}_{2} / \hat{\beta}_{1}\right) \cdot \hat{\beta}_{1}=\hat{\beta}_{2}$.
$0.5 * 40=20$, units of $x_{i 2}$. This situation is shown in Fig. 3 which contains two horizontal axes, the upper one referring to $x_{i 1}$, the lower one to $x_{i 2}$. Both axes are scaled differently in correspondence with their respective influence on $\hat{y}_{i}$.

The next question is how to find the position of a firm with independent variables $x_{i 1}^{*}$ and $x_{i 2}^{*}$ in Fig. 3. Figure 4 presents this situation. Here, both $x_{i 1}^{*}$ and $x_{i 2}^{*}$ are equal to 10 . To find the firm's expected 'normal' value for $\hat{y}_{i}$, we move $x_{i 1}^{*}$, i.e. 10 , along the $x_{1}$-axis, then switch to the $x_{2}$-axis, and move $x_{i 2}^{*}$, i.e. 10 , on this axis. Hereafter, $\hat{y}_{i}$ can be read from the figure: its value is 40 . Of course, the same value of $\hat{y}_{i}$ is obtained if we first move $x_{12}^{*}$ along the $x_{2}$-axis, and then $x_{i 1}^{*}$ on the $x_{1}$-axis. Moreover, many combinations of $x_{1}$ and $x_{2}$ can be found, which lead to the same value of $\hat{y}_{i}$, e.g. the combination $x_{1}=20, x_{2}=5$.

Extension to multi-regressor cases is easy. The main idea is to rescale the axes of all independent variables in correspondence with one particular independent variable. This 'normalizing' variable was chosen to be $x_{1}$ in the case mentioned above. The idea can also be shown in mathematical terms, rewriting expression (2) as:

$$
\hat{y}_{i}=\hat{\beta}_{0}+\left(x_{i 1}+x_{i 2} \cdot\left(\hat{\beta}_{2} / \hat{\beta}_{1}\right)+\cdots+x_{i k} \cdot\left(\hat{\beta}_{\mathrm{k}} / \hat{\beta}_{1}\right)\right) \cdot \hat{\beta}_{1} .
$$




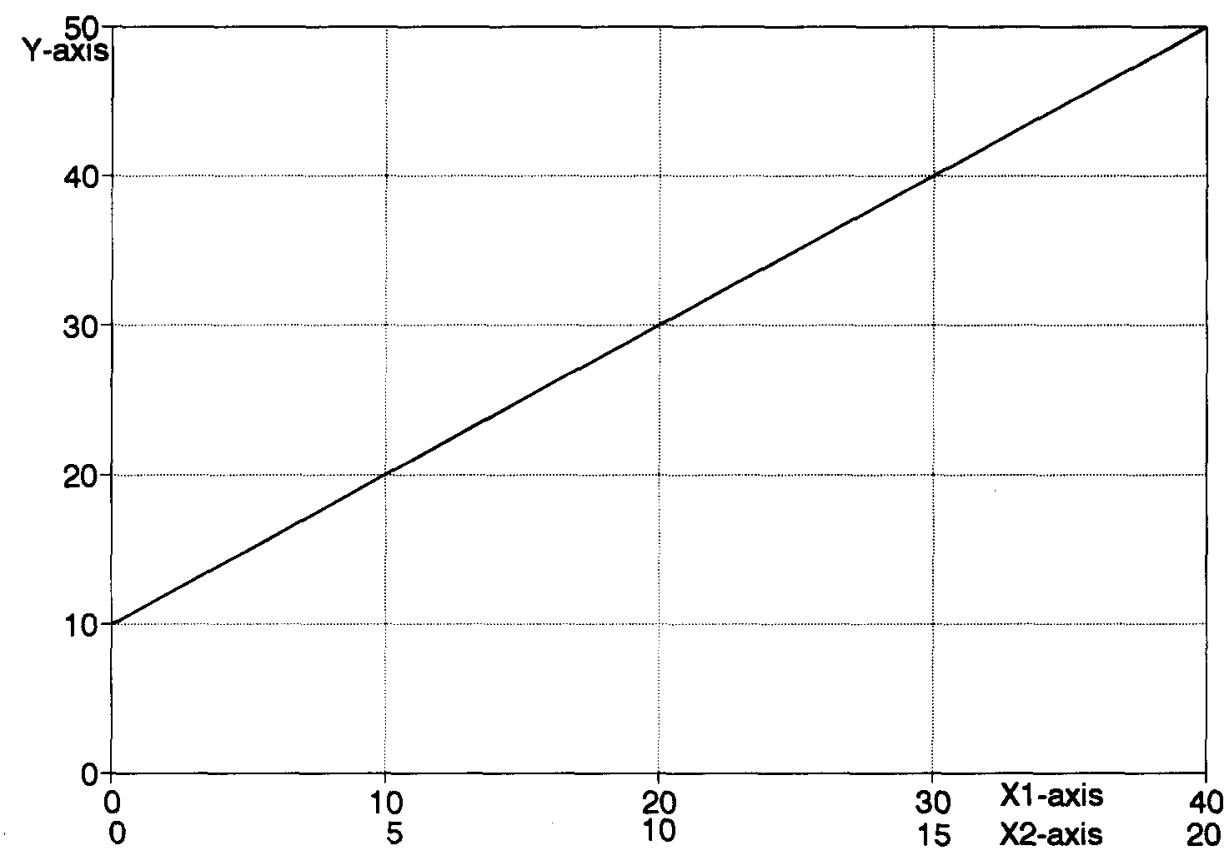

Fig. 3. Plot of $Y$ vs $X 1$ and $X 2$.

It is readily seen, that the multi-regressor case is reduced to a one-regressor case. ${ }^{2}$ However, this one regressor consists of multiple parts: the first part is simply the level of $x_{i 1}$, and the other parts are the rescaled levels of $x_{i j}$, i.e. $x_{i j} *\left(\hat{\beta}_{j} / \hat{\beta}_{1}\right)$ for $j=2, \ldots, k$. These $k$ parts denote the respective contribution of the variables $x_{i 1}$ to $x_{i k}$ to $\hat{y}_{i}$. Of course, if $x_{i 1}$ ranges from 0 to $C$, then $x_{i j}$ ranges from 0 to $C *\left(\hat{\beta}_{1} / \hat{\beta}_{j}\right)$ for $j=1, \ldots, k$.

Using this way to visualize the results obtained with interfirm comparison, it can also in the multi-regressor case be seen which company has the highest ratio, what the ratios of the other companies are and what the expected 'normal' ratios given the characteristics of these companies are. Furthermore, the effect of a change in firm characteristic on the ratio can be read from the horizontal axis corresponding with that firm characteristic. Even more insight is obtained if, below the different axes the values of the characteristics of the companies are drawn, since this indicates the contribution of each firm characteristic to the value of the expected 'normal'

${ }^{2}$ Of course, this expression is only rewritten in order to present the results of the estimation procedure in a neat way. The estimation itself is performed making use of expression (2).

${ }^{3}$ This will especially be the case if the regression is used to analyze observation units, which are of interest themselves, such as well known large firms or industries. ratio. All these applications will be illustrated by a numerical example in Section 4.

\section{Generalizations}

Although, in this paper we focus on the use of the visualization method for interfirm comparison, it will be clear that an abundance of other applications exist. Here, we are thinking of the visualization of all kinds of regressions, where more than one regressor is used. Normally, the parameter estimates, i.e. the $\beta$ 's, seem most important, as they indicate the influence of a particular variable on the explanatory variable.

However, the contribution of a variable to the explanatory variable, i.e. the product of $x_{i j}$ and $\beta_{j}$, might be equally if not more important. ${ }^{3}$ For example, in explaining a firm's performance it might be interesting to know the different contributions of the firm's different products on its performance. Similarly, in explaining an industry's profit margin, it might be interesting to know how much its expenses on research and development contributed to this margin.

\section{A NUMERICAL EXAMPLE}

In this section, a numerical example is presented to illustrate the method discussed in Section 3. The main purpose of the presentation 


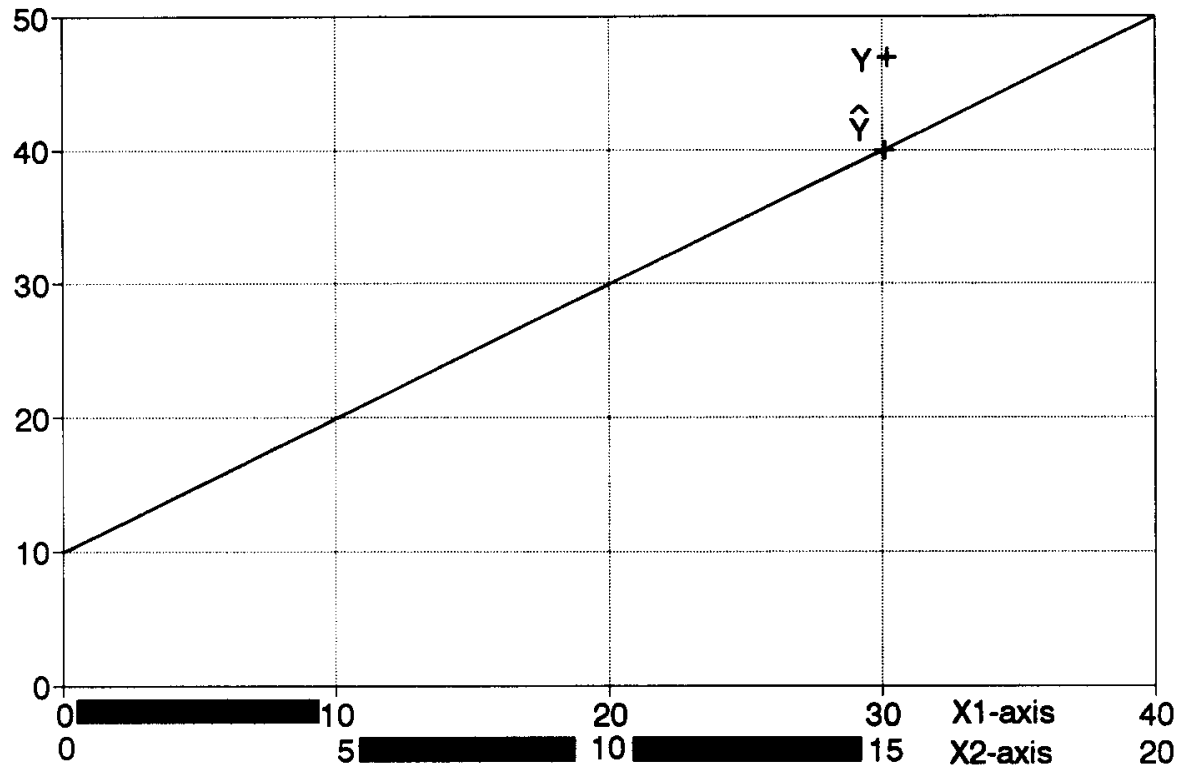

Fig. 4. Interfirm comparison in the two-regressor case.

is to clarify the new visualization method, and not to present the mere estimation results themselves. In other words, the discussion aims to illustrate how the estimation results can be presented compactly making use of figures like Fig. 5.

As part of a multi-factor analysis as described in [1], the following regression relation was estimated making use of data concerning Dutch retail industries:

$$
\mathrm{bcs}_{i}=\beta_{0}+\mathbf{n}_{i} \cdot \beta_{1}+\mathrm{ta}_{i} \cdot \beta_{2}+\mathrm{it}_{i} \cdot \beta_{3}+\epsilon_{i} .
$$

In this relation, $i$ denotes the retail industry, bcs $_{i}$ stands for the business cycle senstivity ${ }^{4}$ of retail industry $i$, and $\mathrm{n}_{i}, \mathrm{ta}_{i}$ and $\mathrm{it}_{i}$ respectively denote indicators ${ }^{5}$ for the number of employees,

${ }^{4}$ The business cycle sensitivity indicates the influence of the business cycle on the cash flow of the firm. If the business cycle is expected to change $x \%$, then the cash flow is expected to change (bcs ${ }_{i}^{*} x$ ). Of course, in reality is it

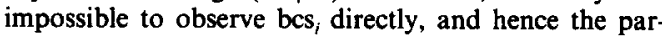
ameters of expression (6) cannot be estimated. For this reason, a larger system of equations was used to estimate the parameters $\beta$. However, as this system is rather complex and of no relevance for the technique presented here, we will not discuss it, and confine ourselves to expression (6), which nicely summarizes the relevant part of the system. More information about the underlying system can be found in [1]

${ }^{5}$ The precise relation between the number of employees, total assets and inventory turnover on the one hand, and their indicators on the other can be found in Appendix B. total assets and inventory turnover; the parameters $\beta$ have to be estimated, and $\epsilon_{i}$ is an error term.

This expression is similar to expressions normally used to explain financial ratios. The only difference is that, here, we explain the sensitivity for a risk factor instead of a financial ratio. Interfirm comparison based on sensitivities can be useful, as it focuses the attention on the risks the firm runs and its future situation.

The following parameter values were estimated: $\hat{\beta}_{0}=4.496 * 10^{-3}, \quad \hat{\beta}_{1}=1.957 * 10^{-4}$, $\hat{\beta}_{2}=6.687 * 10^{-4}$, and $\hat{\beta}_{3}=1.550 * 10^{-5}$. Using these parameter estimates Fig. 5 was drawn. Let us first examine the upper part. The vertical axis refers to the business cycle sensitivity and the horizontal axis to the firm characteristics. The indicator for the number of employees, i.e. $\mathrm{n}_{i}$, was used as 'normalizing' independent variable. Hence, the slope of the line is $\hat{\beta}_{1}=1.957 * 10^{-4}$. Next, the $x$-axes corresponding to $\mathrm{ta}_{i}$ and it were scaled. Using the estimated parameter values, it is computed that 100 units of $n_{i}$ correspond with 29.3 , i.e. $100 *\left(1.957 * 10^{-4}\right)$ $\left.6.687 * 10^{-4}\right)$, units of $\mathrm{ta}_{i}$, and with 1262.2 , i.e. $100 *\left(1.957 * 10^{-4} / 1.550 * 10^{-5}\right)$, units of $i_{i}$.

Apart from the line describing expression (6) the different positions of the retail industries are drawn in Fig. 5. These positions were found making use of Table 1. The first two columns of 

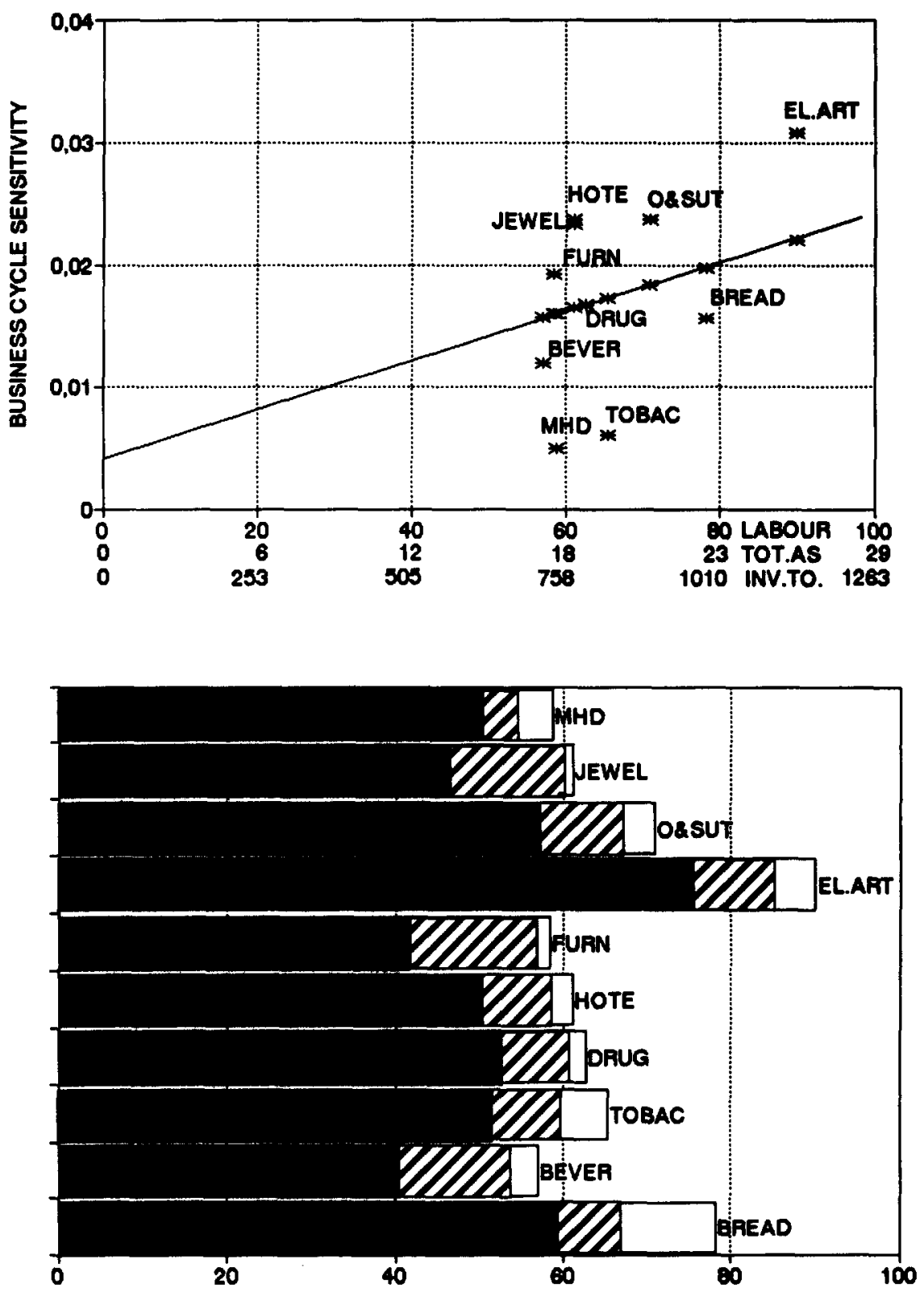

Fig. 5. Visualizing multi-regressor interfirm comparison.

Table 1. Some industry characteristics

\begin{tabular}{llcccccccc}
\hline $\begin{array}{l}\text { SIC- } \\
\text { code }\end{array}$ & Description & $\mathrm{n}$ & $\mathrm{ta}$ & $\mathrm{ta} \cdot\left(\beta_{2} / \beta_{1}\right)$ & $\mathrm{it}$ & $\mathrm{it} \cdot\left(\beta_{3} / \beta_{1}\right)$ & teu & bcs & bcs \\
\hline 6522 & BREAD & 59.79 & 1.94 & 6.62 & 148.15 & 11.73 & 78.14 & 0.0198 & 0.0156 \\
$\mathbf{6 5 2 5}$ & BEVER & 42.84 & 2.70 & 9.24 & 61.61 & 4.88 & 56.96 & 0.0157 & 0.0119 \\
6526 & TOBAC & 51.47 & 2.05 & 7.02 & 86.62 & 6.86 & 65.35 & 0.0173 & 0.0060 \\
6543 & DRUG & 52.25 & 2.53 & 8.63 & 22.09 & 1.75 & 62.63 & 0.0168 & 0.0167 \\
6581 & HOTE & 49.29 & 2.71 & 9.27 & 32.07 & 2.54 & 61.10 & 0.0165 & 0.0238 \\
6591 & FURN & 43.11 & 3.91 & 13.37 & 25.18 & 1.99 & 58.47 & 0.0160 & 0.0192 \\
6595 & EL.ART & 74.50 & 3.55 & 12.11 & 42.91 & 3.40 & 90.02 & 0.0221 & 0.0308 \\
6642 & O\&SUT & 57.13 & 3.20 & 10.95 & 35.37 & 2.80 & 70.88 & 0.0184 & 0.0237 \\
6653 & JEWEL & 47.57 & 3.80 & 12.98 & 7.64 & 0.61 & 61.16 & 0.0165 & 0.0233 \\
9841 & MHD & 52.98 & 0.88 & 2.97 & 34.40 & 2.72 & 58.67 & 0.0160 & 0.0049 \\
\hline
\end{tabular}

Note: $n, t a$ and it respectively denote the indicators for the number of employees, total assets and inventory turnover, teu stands for the total of equivalent units, bês is the expected 'normal' business cycle sensitivity, and bcs the observed business cycle sensitivity. 
Table 1 present the SIC-code and name of 10 retail industries that were investigated. Appendix A provides a clarification of the abbreviations used.

The third column refers to the indicator of the number of employees, $\mathrm{n}_{i}$, and the fourth column to the indicator of total assets, $\mathrm{ta}_{i}$. In order to rescale the latter this indicator was transformed by multiplying by $\left(\hat{\beta}_{2} / \hat{\beta}_{1}\right)$. The result is found in the fifth column. Now, it can be seen that for instance in the case of bakeries (Table 1, first row, columns three and five) the contribution of total assets to the business cycle sensitivity is approximately ten times smaller than the contribution of the number of employees namely 6.62 vs 59.79 .

The sixth column refers to the indicator of inventory turnover, whereas the seventh again is its rescaled analog, which was obtained through multiplying by $\left(\hat{\beta}_{3} / \hat{\beta}_{1}\right)$. It is seen that the influence of inventory turnover on the business cycle sensitivity is approximately twice that of total assets: 11.73 vs 6.62 .

The eighth column, then, presents the total influence of the firm characteristics on the business cycle sensitivity as it is merely the sum of the columns three, five and seven. Column nine presents the corresponding 'normal' business cycle sensitivity, i.e. the sensitivity that would be expected given the level of the firm characteristics. This can be computed by multiplying the eighth column by $\hat{\beta}_{1}$ and adding $\hat{\beta}_{0}$. Finally, the tenth column presents the observed business cycle sensitivity. Concerning bakeries it can be seen that the observed business cycle sensitivity is somewhat lower than its 'normal' business cycle sensitivity: 0.0156 vs 0.0198 .

In the upper part of Fig. 5, the position of the industries were drawn as follows. The vertical coordinate corresponds with the observed business cycle sensitivity, i.e. the tenth column, and the horizontal coordinate with the total influence of the firm characteristics on the business cycle sensitivity, i.e. the eighth column.

The lower part of Fig. 5, the so-called bar chart, describes how the horizontal component of the coordinate was composed by the different firm characteristics. The first block on the bar chart refers to the indicator of the number of employees, the second to the total assets indicator and the third to the inventory turnover indicator. The length of the blocks indicate the contribution of the different firm characteristics to the expected 'normal' value of the business cycle sensitivity. These lengths can be found in the third, fifth and seventh columns of Table 1 for respectively the number of employees indicator, the total assets indicator, and the inventory turnover indicator.

From Fig. 5 it can be seen that the number of employees indicator has by far the most influence on the business cycle senstivity, whereas the influence of the inventory turnover indicator is on average the smallest. It is also seen that the jewellery shops (JEWEL) and the men's hairdressers (MHD) have almost the same level of total firm characteristics: both bars have approximately the same size. However, their compositions differ: the number of employees indicator plays a larger role for the men's hairdressers than for the jewellery shops. On the other had, the total assets indicator of the latter is larger which neutralizes the effect of the lower value of the number of employees indicator. It can also be seen that for the men's hairdressers' inventory turnover plays an important role, whereas this is almost nil for the jewellery shops. Finally, although according to the level of the firm characteristics the expected 'normal' value of the business cycle is nearly the same for both industries, it is seen that the difference between the observed business cycle sensitivities is rather large, see Fig. 5 upper part. The jewellery shops have a higher than (expected) 'normal' business cycle sensitivity in contrast to the men's hairdressers, which have lower than (expected) 'normal' business cycle sensitivity. This difference is not explained by firm characteristics taken into account in our model. In order to find its underlying cause both firms have to be investigated more closely.

\section{SUMMARY AND CONCLUSION}

In this paper, we have presented a method to visualize interfirm comparison techniques. Using this method, it is also possible to visualize the results of interfirm comparison when more than one regressor is used to explain the variable of interest. As a result, insight into interfirm comparison techniques is increased, which is important because these techniques have to be used by managers to whom they have to be intuitively appealing.

The consequences of the new visualization method are that it can also in the multi-regres- 
sor case be seen, which company has the largest sensitivity, what the sensitivities of the other companies are, what the expected sensitivities given the characteristics of these companies are, what the effect of a change in firm characteristic on the sensitivity is, and what the contribution of a certain firm characteristic to the expected 'normal' sensitivity is.

The method was illustrated by a numerical example concerning Dutch retail industries. Apart from the new visualization method, this example contained another new aspect: firms are compared on the basis of their business cycle sensitivity. In other words, interest is not focused on a financial ratio, but on a risk measure. As a consequence, a prospective view on the firm is obtained.

Finally, it should be noted that the applications of this method are not limited to those concerning interfirm comparison. In many regression equations it is interesting to know to what extent the respective independent variables contribute to the explanation of the explanatory variable.

\section{APPENDIX A}

\begin{tabular}{lll}
\hline $\begin{array}{l}\text { SIC- } \\
\text { code }\end{array}$ & Description & Abbreviation \\
\hline 6522 & Bakeries & BREAD \\
6525 & Off-licence shop & BEVER \\
6526 & Tobacco shops & TOBAC \\
6543 & Drugstores & DRUG \\
6581 & Housing textile shops & HOTE \\
6591 & Furniture shops & FURN \\
6595 & Shops selling electronic articles & EL.ART \\
6642 & Shops selling office and school utilities & O\&SUT \\
6653 & Jewelleries & JEWEL \\
9841 & Men's hairdressers & MHD \\
\hline
\end{tabular}

${ }^{6}$ In order to scale the variable inventory turnover, i.e. sales/inventory, it was multiplied by the level of sales. As a result, it can be handled in the same way as the other variables 'No. of hours' and 'Total assets'.

\section{APPENDIX B}

Table B1 contains some more information about the variables used. The relation with Table 1 is as follows. In order to rescale the observations all three firm characteristics, i.e. number of hours, total assets and sales ${ }^{2} /$ inventory, were divided by the level of the cash flow. ${ }^{6}$ Hence, $n$ was computed by dividing the third column into the sixth column, ta by dividing the fourth column into the sixth, and it by dividing the fifth column into the sixth.

Table B1. The industry characteristics

\begin{tabular}{llrccc}
\hline $\begin{array}{c}\text { SIC- } \\
\text { code }\end{array}$ & Description & $\begin{array}{c}\text { No. of } \\
\text { hours }\end{array}$ & $\begin{array}{c}\text { Total } \\
\text { assets }\end{array}$ & $\begin{array}{c}\text { Sales }{ }^{2} \text { inventory } \\
\text { inver }\end{array}$ & Cash flow \\
\hline 6522 & BREAD & 12,905 & 418 & 31,974 & 216 \\
6525 & BEVER & 3804 & 240 & 5471 & 89 \\
6526 & TOBAC & 3954 & 158 & 6654 & 77 \\
6543 & DRUG & 7201 & 348 & 3045 & 138 \\
6581 & HOTE & 7659 & 422 & 4983 & 155 \\
6591 & FURN & 10,216 & 927 & 5966 & 237 \\
6595 & EL.ART & 10,389 & 494 & 5983 & 139 \\
6642 & O\&SUT & 10,607 & 595 & 6568 & 186 \\
6653 & JEWEL & 7675 & 613 & 1232 & 161 \\
9841 & MHD & 4230 & 69 & 2747 & 80 \\
\hline
\end{tabular}

Note: the amounts are in thousands of Dutch guilders.

\section{REFERENCES}

1. Vermeulen EM, Spronk J and van der Wijst D (1993) A multi-factor risk analysis of 18 Dutch industries. Report 9304/F of the Rotterdam Institute for Business Economic Studies, Erasmus University Rotterdam, Rotterdam, The Netherlands.

2. van der Wijst $D$ (1990) Modelling interfirm comparisons in small business. Omega 18, 123-129.

ADDRESS FOR CORRESPONDENCE: Dr EM Vermeulen, Tinbergen Institute and Department of Finance, Erasmus University Rotterdam, PO Box 1738, 3000 DR Rotterdam, The Netherlands. 